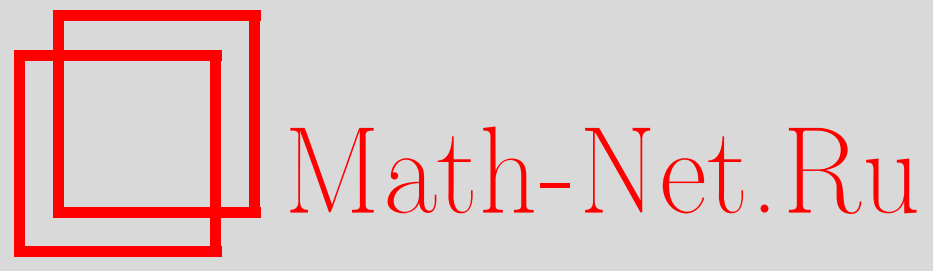

А. В. Хохлов, Нелинейная модель вязкоупругопластичности типа Максвелла: моделирование влияния температуры на кривые деформирования, релаксации и ползучести, Вестн. Сам. гос. техн. унта. Сер. Физ.-мат. науки, 2017, номер 1, 160-179

DOI: https://doi.org/10.14498/vsgtu1524

Использование Общероссийского математического портала MathNet.Ru подразумевает, что вы прочитали и согласны с пользовательским соглашением

http://www . mathnet.ru/rus/agreement

Параметры загрузки:

IP : 54.84 .234 .179

26 апреля 2023 г., 15:50:12

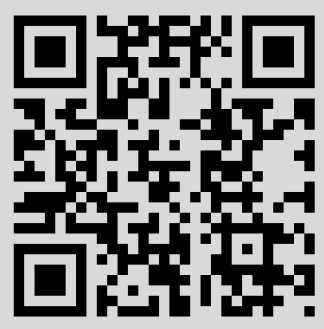


УДК 539.374

\title{
Нелинейная модель вязкоупругопластичности типа Максвелла: моделирование влияния температуры на кривые деформирования, ползучести и релаксации
}

\author{
А. В. Хохлов \\ Московский государственный университет имени М. В. Ломоносова, \\ Научно-исследовательский институт механики, \\ Россия, 119192, Москва, Мичуринский проспект, 1.
}

\section{Аннотация}

Исследуются область применимости, арсенал возможностей и способы идентификации и настройки нелинейного определяющего соотношения типа Максвелла для вязкоупругопластичных материалов с двумя произвольными материальными функциями и двумя параметрами (в случае одноосного изотермического нагружения). Оно нацелено на описание комплекса основных реологических эффектов, типичных для реономных материалов, обладающих наследственностью, высокой скоростной чувствительностью и разносопротивляемостью, для которых характерны установившаяся ползучесть, стадия течения при постоянном напряжении и возрастание податливости, скоростей диссипации, релаксации, ползучести и рэтчетинга и скоростной чувствительности с увеличением температуры. К ним относятся, в частности, многие полимеры, их расплавы и растворы, композиты, твердые топлива, асфальтобетоны, титановые и алюминиевые сплавы, углеродные и керамические материалы при высоких температурах и др.

Для учета влияния температуры на механическое поведение материалов (при изотермических процессах) два материальных параметра модели (коэффициент вязкости и «модуль упругости») рассматриваются как функции температуры. Найдены ограничения на свойства этих функций, необходимые и достаточные для адекватного описания качественных характеристик влияния температуры на экспериментальные кривые ползучести, релаксации, деформирования с постоянными скоростями, ползучести при ступенчатом нагружении, на касательный модуль и предел текучести, скоростную чувствительность и скорость накопления пластической деформации, типичных для стабильных вязкоупругопластичных материалов в изотермических квазистатических испытаниях. Они получены в результате аналитического изучения свойств

\section{Статья}

(2) (7) Контент публикуется на условиях лицензии Creative Commons Attribution 4.0 International (https://creativecommons.org/licenses/by/4.0/deed.ru)

\section{Образец для цитирования}

Хохлов А. В. Нелинейная модель вязкоупругопластичности типа Максвелла: моделирование влияния температуры на кривые деформирования, ползучести и релаксации // Вестн. Сам. гос. техн. ун-та. Сер. Физ.-мат. науки, 2017. Т. 21, № 1. С. 160-179. doi: $10.14498 /$ vsgtu1524.

\section{Сведения об авторе}

Андрей Владимирович Хохлов (1) http://orcid.org/0000-0002-9212-2579

кандидат технических наук; старший научный сотрудник; лаб. упругости и пластичности; e-mail: andrey-khokhlov@ya.ru 
кривых релаксации, ползучести и деформирования, порождаемых определяющим соотношением типа Максвелла с произвольными материальными функциями, и их сопоставления с типичными свойствами экспериментальных кривых реономных материалов. Доказано, что коэффициент вязкости, «модуль упругости» и их отношение (время релаксации ассоциированной линейной модели Максвелла) должны быть убывающими функциями температуры, и это обеспечивает адекватное качественное описание десятка наблюдаемых базовых термомеханических эффектов, свидетельствующих о возрастании податливости материалов (в частности, убывании касательного модуля и предела текучести), ускорении релаксации, ползучести и рэтчетинга и повышении скоростной чувствительности с ростом температуры.

Ключевые слова: вязкоупругопластичность, изотермические условия, влияние температуры, кривые релаксации и ползучести, ступенчатые нагружения, диаграммы деформирования, напряжение течения, скоростная чувствительность, сверхпластичность, рэтчетинг, полимеры.

Получение: 6 декабря 2016 г. / Исправление: 6 марта 2017 г. Принятие: 13 марта 2017 г. / Публикация онлайн:

Введение. Данная статья - продолжение цикла работ, посвященных качественному анализу нелинейного определяющего соотношения (ОС) типа Максвелла для вязкоупругопластичных материалов с целью выявления необходимых ограничений на материальные функции, области применимости, арсенала возможностей и способов идентификации и настройки этого ОС [1-4].

Будем рассматривать изотермические одномерные процессы, характеризуемые в точке тела историей напряжения $\sigma(t)$ и деформации $\varepsilon(t), t>0$. Связь между процессами $\sigma(t)$ и $\varepsilon(t)$ зададим по аналогии с реологической моделью Максвелла, т. е. постулируем, что деформация $\varepsilon(t)$ разлагается в сумму упругой и вязкопластической компонент, каждая из которых зависит от (безразмерного) напряжения $\sigma(t)$, но нелинейно:

$$
\varepsilon=\varepsilon_{e}+\varepsilon_{v}, \quad \varepsilon_{e}=F(\sigma) / E, \quad \dot{\varepsilon}_{v}=V(\sigma) / \eta .
$$

Определяющее соотношение (1) содержит две материальные функции (МФ): $F(x), V(x), x \in\left(\omega_{-}, \omega_{+}\right), \omega_{-}<0, \omega_{+}>0$ (возможны случаи, когда $\omega_{-}=-\infty$, $\left.\omega_{+}=+\infty\right)$ и две константы: «модуль упругости» $E>0$ и коэффициент вязкости $\eta>0$. Если $\omega_{+}$и $\omega_{-}$конечны, то они могут быть интерпретированы как пределы прочности при растяжении и сжатии (как материальные параметры ОС). Процесс $\sigma(t), t \geqslant 0$, в (1) предполагается кусочно-непрерывным и кусочно-гладким (при $t<0$ считаем, что $\sigma \equiv 0)$.

Параметры $E$ и $\eta$ выделены из МФ $F$ и $V$ для удобства сопоставления с линейной моделью Максвелла (получающейся при $V(x)=F(x)=x$ ) и учета влияния температуры в форме $E=E(T), \eta=\eta(T)$. Выявление общих ограничений на качественный характер зависимости от температуры материальных параметров $E$ и $\eta$ в результате анализа зависимостей кривых релаксации, ползучести и деформирования, порожденных ОС (1) с произвольными МФ от $E$ и $\eta$ (а значит, от $T$ ), и является основной целью данной статьи.

Общая тензорная формулировка нелинейных ОС максвелловского типа для (больших деформаций) вязкоупругих сред, родственных ОС (1), описа- 
ние кинематики, термодинамические аспекты и способы конкретизации ОС изучались в работах [5-13]. В [6, 7, 9-13] внимание было сосредоточено на описании поведения жидкостей, авторы этих работ обсуждали эксперименты и эффекты, присущие жидкостям (расплавам и растворам полимеров и т. п.), не рассматривали кривые ползучести, релаксации и деформирования, порождаемые ОС, не задавали многих вопросов, специфичных для механики деформируемого твердого тела, не анализировали соответствующие феноменологические ограничения на материальные функции и параметры ОС (1) и способы их идентификации.

Частный случай $\mathrm{OC}(1)$ с $F(x)=x$ формально совпадает с вырожденным вариантом модели VBO («viscoplasticity based on overstress») [14], когда задано нулевое равновесное напряжение $g(\varepsilon)$. Задав в ОС $(1) F(x)=x$ и $V(x)=x|x|^{n-1}, n>1$, получим трехпараметрическую модель

$$
\dot{\varepsilon}=E^{-1} \dot{\sigma}+\eta^{-1} \sigma|\sigma|^{n-1}
$$

с линейной упругостью и степенной вязкостью. Она во многом близка ОС

$$
\sigma(t)=K \dot{\varepsilon}(t)^{m} \varepsilon(t)^{N}, \quad N \geqslant 0,
$$

традиционно используемому для описания ползучести с деформационным упрочнением и сверхпластического течения [15-21], но теоретические кривые модели (2) лучше и полнее описывают данные испытаний материалов в (пред)сверхпластичном состоянии, и сфера ее применимости гораздо шире, чем сверхпластичность и ползучесть [1-4]. Модель (2) применялась в ряде работ для описания экспериментальных кривых ползучести и решения конкретных задач [15,16,22,23]. В монографии [19] она примерялась к моделированию сверхпластичности. В статьях [24-28] исследовались кривые ползучести, обратной ползучести и деформирования параллельного соединения двух таких моделей с различными показателями $n$, а также модели с $F(x)=x|x|^{m-1}$.

Более подробные обзоры литературы и родственных моделей (в теории ползучести, сверхпластичности и реологии полимеров) приведены в работах $[1,3,4]$.

\section{1. Первичные ограничения на материальные функции нелиней-} ной модели Максвелла. ОС (1) можно записать как в интегральной, так и в дифференциальной формах:

$$
\begin{gathered}
\varepsilon(t)=E^{-1} F(\sigma(t))+\eta^{-1} \int_{0}^{t} V(\sigma(\tau)) d \tau, \quad \text { или } \varepsilon=\Pi \mathbf{\Pi} \sigma, \\
\dot{\varepsilon}=E^{-1} F^{\prime}(\sigma) \dot{\sigma}+\eta^{-1} V(\sigma), \quad t>0 .
\end{gathered}
$$

Материальная функция $F(x)$ в ОС (1) определяет закон упругого деформирования $\varepsilon_{e}=F(\sigma) / E$. Поэтому минимальные первичные ограничения на $F(x)$ (естественные с точки зрения феноменологии и минимальные математические [1,2]) таковы: $F(x), x \in\left(\omega_{-}, \omega_{+}\right),-$непрерывная (строго) возрастающая функция с кусочно-непрерывной производной, такая, что $F(0)=0$ (тогда $x F(x)>0$, т. е. $\operatorname{sgn} F(x)=\operatorname{sgn} x$ ). Последние два условия обеспечивают совпадение знаков $\sigma$ и $\varepsilon_{e}(\sigma)$ и выполнение условия $\varepsilon_{e}(0)=0$. Из строгого возрастания $F(x)$ следует возрастание $\varepsilon_{e}(\sigma)$ и энергии упругой деформации 
с ростом $|\sigma|$, а также существование обратной функции $f=F^{-1}$ на промежутке $(\underline{y}, \bar{y})$, где

$$
\bar{y}:=\sup F(x)=F\left(\omega_{+}-0\right), \quad \underline{y}:=\inf F(x)=F\left(\omega_{-}+0\right) .
$$

В [1] доказано, что МФ $f$ полностью определяет форму диаграммы мгновенного деформирования $\sigma(\varepsilon)$ (предельной кривой, к которой сходятся семейства диаграмм деформирования с постоянной скоростью нагружения или деформирования $\sigma=\sigma(\varepsilon, a)$ и $\sigma(\varepsilon, b)$, когда скорость $a$ или $b$ стремится к бесконечности). Дифференцируемость $F(x)$ требуется для определения скорости упругой и полной деформации в (4) и касательного модуля диаграмм деформирования, порождаемых ОС (1) (см. ниже).

Функция вязкости $V(x) / \eta$ в ОС (1) регулирует наследственные свойства, скорость диссипации, релаксации, ползучести и накопления пластической деформации, чувствительность напряжения к скорости деформации, длительную прочность [1-4]. Чем больше значение $|V(\sigma)| / \eta$, тем меньше вязкое сопротивление (больше $\left|\dot{\varepsilon}_{v}\right|$ и $|\dot{\varepsilon}|$ при том же $\sigma$ ) и тем ближе поведение моделируемого материала к поведению жидкости. Минимальные первичные ограничения на $V(x): V(x)$ - непрерывная (нестрого) возрастающая функция на интервале $\left(\omega_{-}, \omega_{+}\right)$, такая, что $V(0)=0$ (тогда $\left.V(x) x \geqslant 0\right)$.

Исследование кривых релаксации, ползучести и деформирования, порождаемых ОС (1) [1-4], показывает, что следует различать два основных случая, в которых ОС (1) (моделируемый материал) ведет себя по-разному:

1) $|V(x)|>0$ при $x \neq 0$,

2) $V(x) \equiv 0$ на некотором отрезке $\left[\sigma_{-}, \sigma_{+}\right] \subset\left(\omega_{-}, \omega_{+}\right), \sigma_{-} \leqslant 0, \sigma_{+} \geqslant 0$, $\sigma_{+} \neq \sigma_{-}$.

Во втором случае при $\sigma \in\left[\sigma_{-}, \sigma_{+}\right]$OC (1) моделирует (нелинейно) упругое поведение материала (диссипации и гистерезиса нет, релаксация и ползучесть отсутствуют, диаграмма деформирования не зависит от скорости), $\sigma_{-}, \sigma_{+}-$ пределы упругости материала при сжатии и растяжении, а при $\sigma>\sigma_{+}$(или $\left.\sigma<\sigma_{-}\right)$начинают проявляться диссипативные и вязкопластические свойства. В настоящей статье (для краткости) будет рассматриваться только случай $|V(x)|>0$ при $x \neq 0$.

Для материалов с одинаковым поведением при растяжении и сжатии (у которых совпадают как диаграммы деформирования при растяжении и сжатии, так и кривые ползучести и релаксации) МФ должны быть нечетными и $\omega_{-}=-\omega_{+}$. МФ можно задавать лишь при $x \in\left(0, \omega_{+}\right)$и продолжать на $\left(-\omega_{+}, 0\right)$ по формуле $y(x)=y(|x|) \operatorname{sgn}(x)$ (тогда обеспечивается непрерывность производных и потому - единый касательный модуль в нуле, так как $\left.F^{\prime}(0+0)=F^{\prime}(0-0)\right)$. Если материалы при растяжении и сжатии ведут себя не одинаково, то нужно «склеивать» МФ (одну или обе) из двух ветвей на $\left(0, \omega_{+}\right)$и $\left(\omega_{-}, 0\right)$.

K OC (3) применена техника качественного анализа определяющих соотношений для вязкоупругопластических материалов, разработанная ранее в цикле работ $[1-4,29-31]$ и двух десятков других (см. библиографию в работах $[3,4,31])$, посвященных пяти разным ОС. Выведены в общем виде (в виде неявных функций, интегралов и рядов, зависящих от параметров и МФ) уравнения семейств всех основных квазистатических кривых (кривых деформирования, релаксации, ползучести при ступенчатых нагружениях, длительной 
прочности и др.), порождаемых ОС (3), аналитически изучены их качественные свойства при произвольных МФ $F$ и $V$ [1-4]. На основе их сопоставления с набором типичных качественных свойств базовых экспериментальных квазистатических кривых широкого класса вязкоупругопластичных материалов (с целевым списком моделируемых термомеханических эффектов) выведены минимальные необходимые феноменологические ограничения на обе МФ, обеспечивающие адекватное описание экспериментальных кривых, выявлены эффекты, которые ОС (3) принципиально не может описать ни при каких МФ. В данной статье на основе сравнения зависимости свойств теоретических кривых $\mathrm{OC}(3)$ от параметров $E$ и $\eta$ с типичными проявлениями влияния температуры на экспериментальные кривые будут выведены общие качественные ограничения на характер зависимостей $E(T)$ и $\eta(T)$.

Наложенные на МФ ОС (3) в этом пункте первичные ограничения обеспечивают, в частности, термодинамическую согласованность модели, т. е. положительность работы напряжений в произвольном процессе деформирования и неотрицательность диссипации (соблюдение диссипативного неравенства). Действительно, работа напряжений $\sigma(\tau)$ в процессе деформирования $\varepsilon(\tau)$, связанном с $\sigma(\tau)$ соотношением $(4)$, выражается формулой

$$
\begin{gathered}
A=\int_{0}^{t} \sigma(\tau) \dot{\varepsilon}(\tau) d \tau=\int_{0}^{t} E^{-1} \sigma F^{\prime}(\sigma) \dot{\sigma} d \tau+\int_{0}^{t} \eta^{-1} V(\sigma) \sigma d \tau=U+W, \\
U:=E^{-1} \int_{0}^{\sigma} x F^{\prime}(x) d x, \quad W=\eta^{-1} \int_{0}^{t} \sigma(\tau) V(\sigma(\tau)) d \tau .
\end{gathered}
$$

Здесь $U=U(\sigma)$ - энергия упругой деформации, $W=W[t ; \sigma(\tau)]$ - диссипация. Из $F^{\prime}(x)>0$ следует, что $U^{\prime}(\sigma)=E^{-1} \sigma F^{\prime}(\sigma)>0$ при $\sigma>0$ и $U>0$, а из $x V(x)>0$ при $x \neq 0$ следует, что $\dot{W}(t)=\eta^{-1} \sigma(t) V(\sigma(t))>0$ при $\sigma(t) \neq 0$ и $W(t)>0$ при $t>0$. Равенство $W\left(t_{0}\right)=0$ возможно лишь в случае $\sigma(t) \equiv 0$ при $t<t_{0}$ или в случае, когда $V(x) \equiv 0$ на некотором отрезке $\left[\sigma_{-}, \sigma_{+}\right] \subset\left(\omega_{-}, \omega_{+}\right)$, и $\sigma(\tau) \in\left[\sigma_{-}, \sigma_{+}\right]$для всех $\tau<t_{0}$ (т. е. $|\sigma|$ не превышает пределов упругости).

2. Кривые ползучести и релаксации, порождаемые определяющим соотношением (3). При $\sigma(t)=$ const, $t>0$, OC (3) порождает семейство кривых ползучести (КП):

$$
\varepsilon(t, \sigma)=E^{-1} F(\sigma)+\eta^{-1} V(\sigma) t
$$

Если $V(\sigma) \neq 0$, то все КП линейны по времени при $t>0$, т. е. при любых МФ ОС (3) моделирует только ползучесть с постоянной скоростью $\dot{\varepsilon}(t)=\eta^{-1} V(\sigma)$ (как и линейная модель Максвелла), ОС (3) не способно описывать стадии замедленной и ускоренной ползучести, а также ограниченную ползучесть (свойственную, например, многим полимерам). Так как $V(x)>0$ при $x>0$ и $V(x)$ возрастает, то КП (7) возрастает по $t$ (при $\sigma>0$ ) и по $\sigma$, что совпадает с типичными качественными свойствами экспериментальных КП структурно стабильных однородных материалов. Выраженная стадия установившейся ползучести характерна для многих пластичных металлов (в частности, в состоянии сверхпластичности) и полимеров в вязкотекучем состоянии. 
Поскольку в испытаниях стабильных материалов зависимость скорости установившейся ползучести $\dot{\varepsilon}=r(\sigma)$ от напряжения всегда является возрастающей и выпуклой вниз [18,32] при $\sigma>0$, на $V(x)$ необходимо наложить дополнительное ограничение: $V(x)$ долэна быть (нестрого) выпукла вниз при $x>0$ и выпукла вверx при $x<0$ (т. е. $V^{\prime \prime}(x) x \geqslant 0$, если $V^{\prime \prime}(x)$ существует).

В силу (7) МФ $F$ не влияет на скорость ползучести, МФ $V$ не влияет на мгновенную деформацию $\varepsilon(0)=F(\sigma) / E$ и величины скачков $\varepsilon(t)$, соответствующие скачкам $\sigma(t)$. Это позволяет определить $\mathrm{M} \Phi(x) / \eta$ и $F(x) / E$ по отдельности (полностью идентифицировать ОС) по нескольким экспериментальным КП с разными уровнями напряжения (если они качественно сходны с теоретическими КП (7)).

Кривые релаксации ОС $(1)$ - интегральные кривые $\sigma\left(t ; \bar{\varepsilon}, t_{0}\right)$ дифференциального уравнения (4) с заданной деформацией $\varepsilon(t)=\bar{\varepsilon}=$ const при $t \geqslant t_{0}$ :

$$
E^{-1} F^{\prime}(\sigma) \dot{\sigma}+\eta^{-1} V(\sigma)=0, \quad \text { или } \quad \dot{\sigma}=-\tau_{r}^{-1} g(\sigma),
$$

где $t_{0} \geqslant 0$ - продолжительность начальной стадии нагружения («rise time»), т. е. стадии выхода деформации $\varepsilon(t)$ на постоянный уровень $\bar{\varepsilon} \neq 0 ; \tau_{r}=$ $\eta / E>0$ - время релаксации линейной модели Максвелла;

$$
g(x):=V(x) / F^{\prime}(x), \quad x \in\left(\omega_{-}, \omega_{+}\right) .
$$

Для индивидуализации конкретной кривой релаксации $(\mathrm{KP}) \sigma\left(t, \bar{\varepsilon} ; t_{0}, \sigma_{0}\right)$ необходимо помимо значений $\bar{\varepsilon} \neq 0$ и $t_{0} \geqslant 0$ задать начальное значение напряжения $\sigma_{0}=\sigma\left(t_{0}\right) \neq 0$ (так, чтобы $\sigma_{0} \bar{\varepsilon}>0$ ). Если $t_{0}=0$, т. е. деформация считается мгновенной:

$$
\varepsilon(t)=\bar{\varepsilon} h(t)
$$

то $\sigma_{0}=\sigma(0+)$ совпадает с упругим напряжением:

$$
E \bar{\varepsilon}=F\left(\sigma_{0}\right), \quad \text { или } \quad \sigma_{0}=f(E \bar{\varepsilon}), \quad f=F^{-1} \text {. }
$$

Если $t_{0}>0$, то $\sigma_{0}$ зависит от истории $\varepsilon(t)$ при $t \in\left[0 ; t_{0}\right]$ и его вычисление требует использования ОС (3), (4).

Если $|V(\sigma)|>0$, то переменные в (8) разделяются, и можно выписать решение задачи Коши с произвольным начальным условием $\sigma\left(t_{0}\right)=\sigma_{0}>0$ :

$$
t-t_{0}=-\tau_{r} \int_{\sigma_{0}}^{\sigma} \frac{F^{\prime}(x)}{V(x)} d x, \quad t \geqslant t_{0} \quad \text { или } \quad t=t_{0}+\tau_{r} \int_{F(\sigma)}^{F\left(\sigma_{0}\right)} \frac{d y}{V(f(y))}
$$

где $f=F^{-1}$. Уравнение (9) задает семейство $\mathrm{KP} \sigma=R\left(t ; \bar{\varepsilon}, t_{0}\right), t \geqslant t_{0}$, в неявном виде $t=r\left(\sigma ; \bar{\varepsilon}, t_{0}\right)$ (с безразмерным временем $t / \tau_{r}$ ). Параметр $\bar{\varepsilon}$ входит в уравнение $\mathrm{KP}$ через начальное значение $\sigma\left(t_{0}\right)=\sigma_{0}$. Если $t_{0}=0$, то $E \bar{\varepsilon}=F\left(\sigma_{0}\right), \sigma_{0}=f(E \bar{\varepsilon})$, и $\mathrm{KP}(9)$ имеют вид

$$
t=-\tau_{r} \int_{f(E \bar{\varepsilon})}^{\sigma} \frac{F^{\prime}(x)}{V(x)} d x \quad \text { или } \quad t=\tau_{r} \int_{F(\sigma)}^{E \bar{\varepsilon}} \frac{d y}{V(f(y))} .
$$


Анализ неявного представления (9) (и (10)) показывает, что все KP $\sigma=$ $\sigma(t, \bar{\varepsilon})$ с $\bar{\varepsilon}>0$ убывают по $t$ и стремятся $\kappa$ нулю при $t \rightarrow \infty$, a с ростом деформаиии $\bar{\varepsilon}$ смещаются вверх.

$\mathrm{B}$ испытаниях стабильных материалов на релаксацию $\mathrm{KP}$ с $\bar{\varepsilon}>0$ всегда выпуклы вниз, поэтому теоретические $\mathrm{KP} \mathrm{c} \bar{\varepsilon}>0$ (или $\sigma_{0}>0$ ) должны быть выпуклы вниз, т. е. функция $\dot{\sigma}(t)$ должна возрастать $(\ddot{\sigma}(t)>0)$. В силу (8) это равносильно возрастанию функции $g(x):=V(x) / F^{\prime}(x):$ из $(8)$

$$
\ddot{\sigma}(t)=-\tau_{r}^{-1} g^{\prime}(\sigma) \dot{\sigma}=\tau_{r}^{-2} g^{\prime}(\sigma) g(\sigma),
$$

но $g(\sigma)>0$ при $\sigma>0$, и потому $\ddot{\sigma}(t)>0$ равносильно $g^{\prime}(\sigma)>0$. Так как

$$
g^{\prime}(x)=\left[V^{\prime}(x) F^{\prime}(x)-V(x) F^{\prime \prime}(x)\right]\left(F^{\prime}(x)\right)^{-2}
$$

(если существуют $V^{\prime}(x)$ и $\left.F^{\prime \prime}(x)\right)$, неравенство $g^{\prime}(x)>0$ равносильно неравенству

$$
V^{\prime}(x) F^{\prime}(x)-V(x) F^{\prime \prime}(x)>0 .
$$

Таким образом, требование выпуклости вниз всех кривых релаксации $c \bar{\varepsilon}>0$ налагает дополнительное ограничение на $M \Phi$ определяющего соотношения (1) (помимо $F^{\prime \prime}(x) x \geqslant 0$ ):

$$
V^{\prime}(x) / V(x)>F^{\prime \prime}(x) / F^{\prime}(x), \quad x \in\left(0, \omega_{+}\right), \quad \text { т. е. } \quad[\ln V(x)]^{\prime}>\left[\ln F^{\prime}(x)\right]^{\prime} .
$$

Время релаксации нелинейной модели (3) находится из (9) и условия $\sigma\left(\tau+t_{0}\right) / \sigma_{0}=e^{-1}$

$$
\tau=\tau_{r} \int_{\sigma_{0} / e}^{\sigma_{0}} \frac{F^{\prime}(x)}{V(x)} d x=\tau_{r} \int_{F\left(\sigma_{0} / e\right)}^{F\left(\sigma_{0}\right)} \frac{d y}{V(f(y))}
$$

Очевидно, $\tau$ возрастает с ростом $\tau_{r}$ и с убыванием $\sigma_{0}$ :

$$
\tau^{\prime}\left(\sigma_{0}\right)=\tau_{r}\left[g\left(\sigma_{0}\right)^{-1}-g\left(\sigma_{0} / e\right)^{-1}\right]<0,
$$

если выполнен критерий выпуклости КР (11), т. е. $g(x)$ возрастает.

Поведение $\mathrm{KP}(9)$ при $\sigma(t) \rightarrow 0$ (и при $t \rightarrow+\infty$ ) зависит от асимптотики подынтегральной функции $F^{\prime}(x) / V(x)$ при $x \rightarrow 0$ (у нее, вообще говоря, есть особенность в точке $x=0$ из-за условия $V(0)=0$, в частности, особенность есть в случае $\left.F^{\prime}(0+0) \neq 0\right)$. Если интеграл

$$
I\left(\sigma_{0}\right)=-\int_{\sigma_{0}}^{0} \frac{F^{\prime}(x)}{V(x)} d x=\int_{0}^{\sigma_{0}} \frac{F^{\prime}(x)}{V(x)} d x
$$

расходится, то все КР (9) с произвольными $\bar{\varepsilon}, t_{0}, \sigma_{0}$ (как при мгновенном нагружении, так и с начальной стадией деформирования) определены на всем луче $\left[t_{0} ; \infty\right)$ и при $t \rightarrow+\infty$ обладают горизонтальной асимптотой $\sigma=0$. Если же интеграл (13) сходится, то КР с произвольными $\bar{\varepsilon}, t_{0}, \sigma_{0}$ достигает уровня $\sigma=0$ в некоторый момент $t_{*}=t_{0}+\tau_{r} I\left(\sigma_{0}\right)<\infty$, и ОС (3) моделирует материал с полной релаксацией напряжения за конечное время $t_{*}-t_{0}$. 
3. Кривые деформирования при постоянных скоростях нагружения или деформирования. Для программы нагружения $\sigma(t)=b t$ с постоянной скоростью $b>0$ OC (3) дает

$$
\varepsilon(t)=E^{-1} F(b t)+\eta^{-1} \int_{0}^{t} V(b \tau) d \tau .
$$

После замены $x=b \tau, \sigma=b t$ получим уравнение семейства диаграмм деформирования (ДД):

$$
\varepsilon(\sigma, b)=E^{-1} F(\sigma)+(b \eta)^{-1} \int_{0}^{\sigma} V(x) d x, \quad b \sigma>0 .
$$

ДД (14) возрастают по $\sigma$ (т. к. $F(x)$ возрастает и $V(x)>0$ при $x>0$ ) и выпуклы вниз при $b>0$ (т. к. $\partial^{2} \varepsilon / \partial^{2} \sigma=E^{-1} F^{\prime \prime}(\sigma)+\eta^{-1} b^{-1} V^{\prime}(\sigma), F^{\prime \prime}(x)>0$ и $V^{\prime}(x)>0$ ); семейство (14) убывает по $b$ (так как $V(x)>0$ при $x>0$ ). Поэтому ДД в обратной форме $\sigma(\varepsilon, b)$ возрастают по $\varepsilon$, выпуклы вверх на полуоси $\varepsilon>0$ при всех $b>0$, а с ростом $b$ ДД $\sigma(\varepsilon, b)$ смещается вверх (т. е. ОС (3) моделирует положительную скоростную чувствительность материалов).

Для программы деформирования $\varepsilon(t)=a t$ с постоянной скоростью $a>0$ OC (4) дает дифференциальное уравнение для отклика $\sigma(t, a)$ :

$$
\dot{\sigma}=E\left(a-\eta^{-1} V(\sigma)\right) / F^{\prime}(\sigma)
$$

Это уравнение имеет точку равновесия $\sigma=\bar{\sigma}$, где $V(\bar{\sigma})=a \eta, \bar{\sigma}:=v(a \eta)$, $v:=V^{-1}$, и особое решение $\sigma(t)=\bar{\sigma}$, не зависящее от времени $(\bar{\sigma}>0$ для $a>0$, если $V(x)>0$ при $x>0$ ). Прямая $\sigma=\bar{\sigma}$ разбивает квадрант $t \geqslant 0$, $\sigma \geqslant 0$ на две области; интегральные кривые уравнения (15), лежащие ниже этой прямой (в частности, интересующее нас решение с начальным условием $\sigma(0)=0)$, обладают свойством $\dot{\sigma}>0$ при $t>0$, т. е. возрастают, а интегральные кривые, лежащие выше этой прямой, обладают свойством $\dot{\sigma}<0$ при $t>0$ (убывают). Все они не могут пересечь прямую $\sigma=\bar{\sigma}$ по теореме единственности решения задачи Коши. В силу (15) по мере приближения к особому решению $\sigma=\bar{\sigma}$ по любой траектории выполняется $\dot{\sigma}(t) \rightarrow 0$.

При $\sigma \neq \bar{\sigma}$ можно разделить переменные в (15) и проинтегрировать:

$$
\frac{F^{\prime}(\sigma) d \sigma}{a \eta-V(\sigma)}=E \eta^{-1} d t, \quad \int_{\sigma_{0}}^{\sigma} \frac{F^{\prime}(x) d x}{a \eta-V(x)}=E \eta^{-1} t, \quad \sigma_{0}:=\sigma(0) .
$$

Подставив $t=\varepsilon / a$ в решение с начальным значением $\sigma_{0}=0$, получим уравнение семейства ДД при постоянной скорости деформирования $a$ в виде $\varepsilon=\varepsilon(\sigma, a)$ :

$$
\varepsilon(\sigma, a)=E^{-1} a \eta \int_{0}^{\sigma} \frac{F^{\prime}(x) d x}{a \eta-V(x)},
$$

или

$$
\varepsilon(\sigma, a)=E^{-1} \int_{0}^{F(\sigma)} \frac{d y}{1-(a \eta)^{-1} V(f(y))}, \quad a \sigma>0,
$$

где $f=F^{-1}$. Производная $F^{\prime}(x)$, вообще говоря, может иметь (интегрируемую) особенность при $x=0$ (например, в случае степенной $М \Phi F(x)=x^{N}$ c $N \in(0,1))$. 
Анализ представления (16) показал [2], что при любых допустимых МФ (см. п. 1) и любых $\eta, E>0, a>0$ ДД (16) определена только при $\sigma \in[0, \bar{\sigma})$, где $\bar{\sigma}:=v(a \eta)$, имеет асимптоту $\sigma=\bar{\sigma}$ (параллельную оси $\varepsilon$ ), монотонно возрастает и выпукла вниз, аналитична в $(0, \bar{\sigma})$, дважды дифференцируема на $[0, \bar{\sigma})$ и имеет при $\sigma \rightarrow 0$ асимптотику:

$$
\varepsilon=F(\sigma) / E+O\left(F(\sigma)^{2}\right) .
$$

Отсюда следует, что обратная функция, т. е. любая ДД в форме $\sigma=\sigma(\varepsilon, a)$, возрастает по $\varepsilon$ выпукла вверх при $\varepsilon>0(u a>0) ;$ с ростом а ДД смещается вверх; каждая ДД $\sigma(\varepsilon, a)$ имеет при $\varepsilon \rightarrow 0$ асимптотику

$$
\sigma=f(E \varepsilon)+O\left(f(E \varepsilon)^{2}\right)
$$

не зависящую от $a$, а при $\varepsilon \rightarrow \infty$ обладает горизонталъной асимптотой $\sigma=\bar{\sigma}$, где $\bar{\sigma}=v(a \eta)$. Последнее свойство означает, что ОС (3) моделирует длительное течение при постоянном напряжении $\bar{\sigma}=\bar{\sigma}(a)$, характерное, в частности, для полимеров в вязкотекучем состоянии и материалов в режимах сверхпластичности. Таким образом, значение $\bar{\sigma}$ можно интерпретировать как оценку сверху для предела текучести и предела прочности моделируемого материала (в случае их зависимости от скорости деформации). Так как $v(0)=0$, то $\bar{\sigma}(a) \rightarrow 0$ при $a \rightarrow 0$, т. е. при малых скоростях материал ведет себя подобно жидкости: течет при малом напряжении (но время выхода ДД на асимптоту $\sigma=\bar{\sigma}$ и соответствующая деформация могут быть довольно большими).

Асимптотика при $\sigma \rightarrow 0 \pm 0$ любой ДД (14) или (16) не зависит от скоростей деформирования или нагружения и совпадает с асимптотикой МФ $F(\sigma) / E$ (скорости $a, b$ и МФ $V$ влияют лишь на $\varepsilon^{\prime \prime}(0, a)$ и $\varepsilon^{\prime \prime}(0, b)$, т. е. на член порядка $\left.O\left(\sigma^{2}\right)\right)$. Соответственно, для любой ДД в борме $\sigma=\sigma(\varepsilon, a)$ $u \sigma(\varepsilon, b)$ касательный модуль в точке $\varepsilon=0$ равен $E / F^{\prime}(0 \pm 0)$ и не зависит om скоростей a u b (зависит только от их знака, если $F^{\prime}(0+0) \neq F^{\prime}(0-0)$, т. е. в случае моделирования разномодульного материала).

При $а \rightarrow \infty$ семейство ДД (16) сходится $\kappa$ кривой (мгновенного дебормирования) $\sigma=f(E \varepsilon)$ [1], равномерно на любом отрезке оси $\varepsilon$ (обратная функция $f(y)$ выпукла вверх при $y>0$, так как $F(x)$ возрастает и выпукла вверх). K этой же кривой сходятся (снизу) и семейство ДД (14) при $b \rightarrow \infty$, и семейство изохронных кривых ползучести (7) при $t \rightarrow 0$. Для всех ДД верна оценка $|\sigma| \leqslant f(E \varepsilon)$ (для любых $a, b$ и $\varepsilon$ ); если $V(x)>0$ в проколотой окрестности $x=0$, то выполняется строгое неравенство $|\sigma|<f(E \varepsilon)$ для всех $\varepsilon \neq 0$.

4. Кривые ползучести при ступенчатых нагружениях, дрейф мгновенно-упругой деформации вследствие ползучести и рэтчетинг. Рассмотрим программу нагружения из $n$ ступеней:

$$
\sigma(t)=\sum_{i=1}^{n-1} \sigma_{i}\left[h\left(t-t_{i-1}\right)-h\left(t-t_{i}\right)\right]+\sigma_{n} h\left(t-t_{n-1}\right)
$$

с произвольными $\sigma_{i} \in\left(\omega_{-}, \omega_{+}\right)$и $t_{i}>0\left(t_{0}=0, t_{i}>t_{i-1}, i=1, \ldots, n-1\right.$, и $\sigma(t)=\sigma_{n}$ при $\left.t>t_{n-1}\right)$. В силу $(3)$ отклик $\varepsilon(t)-$ кусочно-линейная функция 
с разрывами в точках $t=t_{i}[3]$ :

$$
\begin{gathered}
\varepsilon(t)=E^{-1}\left[V\left(\sigma_{i}\right) \tau_{r}^{-1}\left(t-t_{i-1}\right)+F\left(\sigma_{i}\right)\right]+p_{i-1}, t \in\left(t_{i-1}, t_{i}\right), i=1, \ldots, n, \\
p_{i-1}:=\eta^{-1} \sum_{m=1}^{i-1} V\left(\sigma_{m}\right)\left(t_{m}-t_{m-1}\right), \quad i=2, \ldots, n
\end{gathered}
$$

Накопленная пластическая деформация (18) выражает влияние предыдущих $(i-1)$ ступеней нагружения $\left(p_{0}:=0\right)$. Она не зависит от порядка приложения ступеней нагрузки. Скорости ползучести и диссипации постоянны на каждом интервале $\left(t_{i-1}, t_{i}\right)$ :

$$
\dot{\varepsilon}(t)=E^{-1} V\left(\sigma_{i}\right) \tau_{r}^{-1}=\eta^{-1} V\left(\sigma_{i}\right), \quad \dot{W}(t)=\eta^{-1} \sigma_{i} V\left(\sigma_{i}\right)>0 .
$$

Скачки деформации в точках $t=t_{i}$, порожденные скачками напряжения $\sigma_{i+1}-\sigma_{i}$, равны $\left[F\left(\sigma_{i+1}\right)-F\left(\sigma_{i}\right)\right] / E$, скачки скорости ползучести $\dot{\varepsilon}(t)$ равны $\left[V\left(\sigma_{i+1}\right)-V\left(\sigma_{i}\right)\right] / \eta$. Эти скачки не зависят от момента времени $t_{i}$ и предыстории нагружения, а только от $\sigma_{i}$ и скачка напряжения $\sigma_{i+1}-\sigma_{i}$. Для любой МФ $F$ скачок деформации (полной или упругой) при разгрузке (от $\sigma_{i+1}$ до $\sigma_{i}$ ) всегда равен по модулю скачку при догрузке (от $\sigma_{i}$ до $\sigma_{i+1}$ ). Поэтому нелинейное ОС (3) (как и линейное ОС вязкоупругости с произвольной функцией ползучести) не способно моделировать явление «дрейфа мгновенно-упругой дебормации вследствие дебормации ползучести» [26-28, 33, 34-36]. В работе [37] установлено, что нелинейное ОС наследственности, предложенное Ю. Н. Работновым [15,38], описывает явление дрейфа (скачок деформации при разгрузке отличается по модулю от скачка при догрузке, так как зависит от предыстории нагружения).

В работе [3] доказано, что ОС (3) воспроизводит эффект Кольрауша (он наблюдается у полимеров при трехступенчатых программах нагружения с $\left.\sigma_{1}>0, \sigma_{2}<0, \sigma_{3}=0\right)$ тогда и только тогда, когда выполнена система неравенств

$$
0<V\left(\sigma_{2}\right)\left(t_{2}-t_{1}\right)+V\left(\sigma_{1}\right) t_{1}<-\tau_{r} F\left(\sigma_{2}\right)
$$

При периодическом ступенчатом нагружении с двумя ступенями равной длительности $0.5 \tau$ в цикле $\left(t_{i+1}-t_{i}=t_{1}=0.5 \tau, \sigma_{2 i+1}=\sigma_{1}, \sigma_{2 i}=\sigma_{2}, i=1, \ldots\right)$ на интервалах $t \in\left(t_{i-1}, t_{i}\right)$ справедливы формулы $(17),(18)$, а приращение пластической деформации за $j$-тый цикл выражается формулой

$$
p_{2 j}-p_{2(j-1)}=0.5 \tau \eta^{-1}\left[V\left(\sigma_{2 j-1}\right)+V\left(\sigma_{2 j-2}\right)\right]=0.5 \tau \eta^{-1}\left[V\left(\sigma_{1}\right)+V\left(\sigma_{2}\right)\right] .
$$

Если $V(x) \neq 0$ при $x \neq 0$, то несимметричное циклическое нагружение $\left(\right.$ с $\left.\sigma_{2} \neq-\sigma_{1}\right)$ всегда вызывает неограниченное нарастание модуля пластической деформации («ratcheting») с постоянной скоростью (20) за цикл. Исключение составляют специальные программы со свойством $V\left(\sigma_{2}\right)=-V\left(\sigma_{1}\right)$. Если МФ $V(x)$ нечетна, то любое количество симметричных циклов нагружения $\left(\sigma_{2}=-\sigma_{1}\right)$ дает нулевую пластическую деформацию, а КП (17) периодическая функция с периодом $\tau$. Если же диссипативные свойства материала и скорости ползучести при растяжении и сжатии не одинаковы $(V(x)$ не нечетна), то рэтчетинг происходит и при $\sigma_{2}=-\sigma_{1}$. 
Таким образом, ОС (3) описывает рэтчетинг без стабилизации и приспособляемости, т. е. поведение циклически разупрочняющихся материалов.

5. Характер зависимости материальных параметров модели (3) от температуры и ее влияние на теоретические кривые релаксации, ползучести и деформирования. Материальные параметры ОС (3) зависят от температуры $T: E=E(T)$ и $\eta=\eta(T)$ (удобное описание зависимости от $T$ - одна из причин выделения скалярных множителей $E$ и $\eta$ из МФ $F(x)$ и $V(x))$. В квазистатических испытаниях большинства стабильных материалов (в которых не происходят химические, фазовые и структурные превращения) с ростом температуры наблюдается смещение вниз кривых релаксации и смещение вверх кривых ползучести, увеличение скоростей ползучести и релаксации, смещение вниз всех диаграмм деформирования (ДД) $\sigma(\varepsilon, a)$ и $\sigma(\varepsilon, b)$ с постоянными скоростями деформирования или нагружения, уменьшение мгновенного модуля и предела текучести (если есть площадка текучести на ДД).

Выясним, какие ограничения надо наложить на зависимости $E=E(T)$ и $\eta=\eta(T)$, чтобы теоретические кривые $(7),(9),(14),(16),(17)$ адекватно описывали влияние температуры, наблюдаемое в изотермических квазистатических испытаниях.

В силу (7) скорость ползучести $\dot{\varepsilon}(t)=V(\sigma) / \eta$ убывает по $\eta$, потому критерием увеличения скорости ползучести с ростом температуры является убъвание коэбфиииента вязкости $\eta(T)$. Оно обеспечивает смещение вверх всех кривых ползучести (7), возрастание скорости диссипации $\dot{W}(t)=\sigma V(\sigma) / \eta$, скачков скорости ползучести $\left[V\left(\sigma_{i+1}\right)-V\left(\sigma_{i}\right)\right] / \eta$, порожденных скачками напряжения (см. (17)), и накопленной пластической деформации (18), а также ускорение рэтчетинга при циклических нагружениях (в силу (20)). Кроме того, смещение вверх начального значения кривой ползучести (7) обеспечивается убыванием $E(T)$; тогда с ростом температуры возрастает и чувствительность кривых ползучести (17) к скачкам напряжения (скачки деформации $\left[F\left(\sigma_{i+1}\right)-F\left(\sigma_{i}\right)\right] / E$ возрастают).

Убъвание функиии $E(T)$ необходимо и достаточно для убывания касательного модуля

$$
\left.\sigma_{\varepsilon}^{\prime}(\varepsilon, a)\right|_{\varepsilon= \pm 0}=E / F^{\prime}(0 \pm 0)
$$

любой ДД $\sigma=\sigma(\varepsilon, a)$ в точке $\varepsilon=0$ (при растяжении и сжатии) и для смещения вниз всей кривой мгновенного деформирования $\sigma(\varepsilon, \infty)=f(E \varepsilon)$ с ростом температуры (в силу возрастания функции $f:=F^{-1}$ ). Нестрогое убывание $E(T)$ и убывание $\eta(T)$ влекут смещение вниз (на интервале $\varepsilon>0$ ) любой ДД $\sigma=\sigma(\varepsilon, a)$ и $\sigma(\varepsilon, b)$ с постоянной скоростью деформирования или нагружения, заданной (в неявной форме) уравнениями (16) и (14). Это следует из (16) и (14), так как деформации $\varepsilon=\varepsilon(\sigma, a)$ и $\varepsilon=\varepsilon(\sigma, b)$ убывают с ростом $E$ и $\eta$ и, следовательно, увеличиваются как с убыванием $E(T)$, так и с убыванием $\eta(T)$ при любых фиксированных $\sigma, a$ и $b$ (их влияния на ДЛ (16) и (14) сонаправлены). Напряжение течения $\bar{\sigma}=v(a \eta)$ при постоянной скорости деформирования (каждая ДД $\sigma(\varepsilon, a)$ имеет горизонтальную асимптоту $\sigma=\bar{\sigma}$ ) понижается с ростом температуры, так как МФ $v=V^{-1}$ возрастает, а $\eta(T)$ убывает. Именно это и наблюдается при испытаниях подавляющего большинства стабильных вязкоупругопластичных материалов. Убывание $E(T)$ и $\eta(T)$ 
приводит к возрастанию податливости (уменьшению жесткости), к уменьшению касательного модуля, а также к возрастанию энергии деформации (6) и скорости диссипации

$$
\dot{W}(t)=\sigma(t) V(\sigma(t)) / \eta
$$

при любой фиксированной программе нагружения $\sigma(t)$.

Из анализа свойств кривых релаксации (9) следует еще одно необходимое ограничение на зависимости $E(T)$ и $\eta(T)$. По (8) модуль скорости релаксации пропорционален параметру $\tau_{r}^{-1}=E / \eta$. С ростом температуры кривые релаксации $\sigma(t ; \bar{\varepsilon})$ с фиксированным $\bar{\varepsilon}$ (т. е. $\sigma_{0}$ ) должны быстрее сближаться с осью $\sigma=0$ (как и экспериментальные кривые), и потому функиия $\tau_{r}(T)=\eta / E$ должна убывать, т. е. $\eta(T)$ должна убывать быстрее, чем $E(T)$ :

$$
E(T) \eta^{\prime}(T)-E^{\prime}(T) \eta(T)<0, \quad \text { или } \quad E^{\prime}(T) / E(T)>\eta^{\prime}(T) / \eta(T) .
$$

В испытаниях большинства материалов, например, у полимеров в стекловидном и высокоэластичном состояниях и у металлов и сплавов как раз наблюдается более сильная чувствительность вязкости (и предела текучести) к изменению температуры, чем модуля Юнга [39-44]. Убывание функции $\tau_{r}(T)$ равносильно убыванию времени релаксации (12) модели (3) с ростом $T$. Из убывания $\tau_{r}(T)$ следует также, что область (19) в пространстве параметров $\left(t_{1}, t_{2}, \sigma_{1}, \sigma_{2}\right)$ трехступенчатой программы нагружения (с $n=3$ и $\left.\sigma_{3}=0\right)$, обеспечивающая воспроизведение эффекта Кольрауша моделью (3), сужается с ростом $T$.

Таким образом, доказано: чтобы теоретические кривые деформирования, релаксации и ползучести ОС (3) вели себя при изменении температуры так же, как и кривые изотермических испытаний большинства стабильных вязкоупругопластичных материалов, необходимо (и достаточно), чтобъ $E$ u $\eta$, a также $\tau_{r}=\eta / E$, были убъвающими функииями $T$ (или гомологической температуры, или отношения к температуре стеклования для полимеров).

Для обеспечения убывания $E(T), \eta(T)$ и $\tau_{r}(T)$ можно, например, задать их в виде

$$
E=E_{0} \exp (u / T), \quad \eta=\eta_{0} \exp (w / T), \quad w>u>0 .
$$

Тогда время релаксации и скорость ползучести выражаются формулами

$$
\begin{aligned}
& \tau_{r}(T)=\eta_{0} E_{0}^{-1} \exp ((w-u) / T), \quad w-u>0 \\
& \dot{\varepsilon}(t)=V(\bar{\sigma}) / \eta=\eta_{0}^{-1} V(\bar{\sigma}) \exp (-w / T)
\end{aligned}
$$

Скорость ползучести будет возрастать по $T$, а время релаксации будет убывать. Именно в экспоненциальной форме $(21)$ (и с экспоненциальной $V(\bar{\sigma})$ ) обычно принимают их зависимость от температуры в работах по ползучести и длительной прочности (по аналогии с законом Аррениуса для кинетических процессов) [15-21, 39-46].

Заключение. В статье продолжено аналитическое исследование нелинейного определяющего соотношения типа Максвелла (3) для вязкоупругопластичных разносопротивляющихся материалов. Одна материальная функция ОС (3) управляет (нелинейно) упругими свойствами, вторая - вязкопластическими: она регулирует наследственные свойства, вязкость, скорость диссипации, чувствительность напряжения (в частности, мгновенного модуля 
и предела текучести) к скорости деформации, зависимость скорости ползучести и релаксации от величины и знака напряжения и накопление пластической (необратимой) деформации. Для учета влияния температуры на механическое поведение материалов (при изотермических процессах) два материальных параметра модели (коэффициент вязкости и «модуль упругости») рассматриваются как функции температуры.

В результате сопоставления обнаруженных свойств теоретических кривых ползучести, релаксации и деформирования (7), (9), (14), (16), (17), порождаемых $\mathrm{OC}(3)$, с типичными свойствами кривых квазистатических испытаний вязкоупругопластичных материалов выявлены качественные ограничения на характер зависимости двух параметров ОС (3) от температуры, необходимые и достаточные для качественного описания типичных результатов изотермических испытаний широкого класса стабильных реономных материалов на релаксацию, деформирование или нагружение с постоянной скоростью, ползучесть при любых ступенчатых нагружениях и усталость. При минимальных первичных ограничениях на две материальные функции ОС (3) доказано, что для того, чтобы теоретические кривые релаксации (9), ползучести (7), (17) и деформирования (14), (16), порождаемые ОС (3), вели себя при изменении температуры так же, как и экспериментальные кривые большинства стабильных вязкоупругопластичных материалов, необходимо, чтобъ параметры $E$, $\eta$ и $\tau_{r}=\eta / E$ были убъвающими функииями температуры (или гомологической температуры, или отношения к температуре стеклования для полимеров).

Из убывания функций $E(T), \eta(T)$ и $\tau_{r}(T)$ следует, что рост температуры вызывает следующие основные изменения в поведении модели (3):

1) теоретическая скорость диссипации $\dot{W}(t)=\sigma(t) V(\sigma(t)) / \eta$ и работа (5) напряжения $\sigma(\tau)$ в процессе деформирования (как при ползучести, так и при любом нагружении $\sigma(\tau), \tau<t$ ) возрастают;

2) кривые ползучести ОС (3) с $\sigma>0$ смещаются вверх;

3 ) кривые релаксации и диаграммы деформирования $\sigma=\sigma(\varepsilon, a)$ и $\sigma(\varepsilon, b)$, $\varepsilon>0$, при постоянных скоростях деформации $\dot{\varepsilon}=a$ или нагружения $\dot{\sigma}=b$, смещаются вниз;

4) напряжение течения и мгновенный модуль упругости (при любой фиксированной скорости деформирования $а$ ) понижаются;

5) скорости релаксации и ползучести возрастают;

6) модули скачков деформации и ее скорости, порожденные скачками напряжения, увеличиваются (но не зависят от знака скачка напряжения);

7) накопленная пластическая (необратимая) деформация (18) возрастает;

8) рэтчетинг при циклических нагружениях ускоряется;

9) область (19) в пространстве параметров трехступенчатой программы нагружения, обеспечивающая воспроизведение эффекта Кольрауша, сужается.

Таким образом, требование убывания функций $E(T), \eta(T)$ и $\tau_{r}(T)$ обеспечивает адекватное качественное описание полутора десятков наблюдаемых в испытаниях базовых термомеханических эффектов, свидетельствующих о возрастании податливости, скорости диссипации и скоростной чувствительности материалов с ростом температуры.

Проведенный анализ показывает, что нелинейное ОС (3) может приме- 
няться для описания комплекса основных реологических эффектов, типичных для вязкоупругопластичных материалов, обладающих наследственностью, высокой (положительной) чувствительностью к скорости деформирования и, возможно, разносопротивляемостью, для которых характерны стадия установившей ползучести, выраженная «площадка текучести» на диаграммах деформирования с постоянной скоростью, (возрастающая) зависимость предела текучести от скорости деформирования, «неограниченное» нарастание пластической деформации при циклическом нагружении за пределом упругости (рэтчетинг без стабилизации и приспособляемости, циклическое разупрочнение) и возрастание скоростей диссипации, релаксации и ползучести, податливости и скоростной чувствительности с увеличением температуры. Подобное поведение демонстрируют многие полимеры, их расплавы и растворы, композиты, твердые топлива, асфальтобетоны, титановые и алюминиевые сплавы, углеродные и керамические материалы при высоких температурах. В частности, ОС (3) и его модификации могут быть полезны и для моделирования материалов в режимах сверхпластического деформирования.

Конкурирующие интересы. У меня нет конкурирующих интересов.

Авторская ответственность. Я несу полную ответственность за предоставление окончательной версии рукописи в печать. Окончательная версия рукописи мною одобрена.

Финансирование. Работа выполнена при поддержке РФФИ (проект № 17-0801146 _a).

\section{Библиографический список}

1. Хохлов А. В. Свойства семейства диаграмм деформирования с постоянной скоростью нагружения, порождаемых нелинейной моделью вязкоупругопластичности типа Максвелла// Машиностроение и инженерное образование, 2017. №1. С. 14-28.

2. Хохлов А. В. Свойства нелинейной модели вязкоупругопластичности типа Максвелла с двумя материальными функциями // Вестник Московского университета. Сер. 1. математика, механика, 2016. №6. С. 36-41.

3. Хохлов А. В. Нелинейная модель вязкоупругопластичности типа Максвелла: свойства кривых ползучести при ступенчатых нагружениях и условия накопления пластической деформации // Машиностроение и инженерное образование, 2016. № 3. С. 35-48.

4. Хохлов А. В. Кривые длительной прочности нелинейной модели вязкоупругопластичности типа Максвелла и правило суммирования поврежденности при ступенчатых нагружениях // Вестн. Сам. гос. техн. ун-та. Сер. Физ.-мат. науки, 2016. Т. 20, № 3 . C. 524-543. doi: 10.14498/vsgtu1512.

5. Ильюшин А. А., Огибалов П. М. Некоторое обобщение моделей Фойгта и Максвелла // Механика полимеров, 1966. № 2. С. 190-196.

6. Городцов В. А., Леонов А. И. О кинематике, неравновесной термодинамике и реологических соотношениях в нелинейной теории вязкоупругости // ПMМ, 1968. Т. 32, № 1 . C. $70-94$.

7. Leonov A. I., Lipkina E. Ch., Paskhin E. D., Prokunin A. N. Theoretical and experimental investigations of shearing in elastic polymer liquids // Rheol. Acta, 1976. vol. 15, no.7/8. pp. 411-426. doi : 10.1007/BF01574496.

8. Пальмов В. А. Реологические модели в нелинейной механике деформируемых тел// Успехи механики, 1980. Т. 3, № 3. С. 75-115.

9. Прокунин А. Н. О нелинейных определяющих соотношениях максвелловского типа для описания движения полимерных жидкостей // ПММ, 1984. Т. 48, №6. С. 957-965. 
10. Larson R. G. Constitutive Equations for Polymer Melts and Solutions. Boston: Butterworth, 1988. 364 pp. doi: 10.1016/c2013-0-04284-3.

11. Leonov A. I. Analysis of simple constitutive equations for viscoelastic liquids // Journal of Non-Newtonian Fluid Mechanics, 1992. vol.42, no.3. pp. 323-350. doi:10.1016/ 0377-0257 (92) 87017-6.

12. Leonov A. I., Prokunin A. N. Non-linear Phenomena in Flows of Viscoelastic Polymer Fluids. London: Chapman and Hall, 1994. xvii+475 pp. doi : 10.1007/978-94-011-1258-1.

13. Leonov A. I. Constitutive equations for viscoelastic liquids: Formulation, analysis and comparison with data// Rheology Series, 1999. vol.8. pp. 519-575. doi:10.1016/ S0169-3107 (99) 80040-9.

14. Kremple E., Ho K. Inelastic Compressible and Incompressible, Isotropic, Small Strain Viscoplasticity Theory Based on Overstress (VBO) / Handbook of Materials Behavior Models. New York: Academic Press, 2001. pp. 336-348. doi : 10.1016/b978-012443341-0/50037-5.

15. Работнов Ю. Н. Ползучесть элементов конструкиий. М.: Наука, 1966. 752 с.

16. Малинин Н. Н. Расчеты на ползучесть элементов машиностроительных конструкций. М.: Машиностроение, 1981. 221 с.

17. Betten J. Creep Mechanics. Berlin, Heidelberg: Springer-Verlag, 2008. xiii+353 pp. doi : 10 . $1007 / \mathrm{b} 138749$.

18. Локощенко А. М. Ползучесть и длительная прочность металлов. М.: Физматлит, 2016. 504 c.

19. Васин Р. А., Еникеев Ф. У. Введение в механику сверхпластичности. Уфа: Гилем, 1998. $280 \mathrm{c}$.

20. Nieh T. G., Wadsworth J., Sherby O. D. Superplasticity in metals and ceramics / Cambridge Solid State Science Series. Cambridge: Cambridge University Press, 1997. xiv +273 pp. doi : 10.1017/CB09780511525230.

21. Segal V. M., Beyerlein I. J., Tome C. N., Chuvil'deev V. N., Kopylov V. I. Fundamentals and Engineering of Severe Plastic Deformation. New York: Nova Science Pub. Inc., 2010. $542 \mathrm{pp}$.

22. Cao Y. Determination of the creep exponent of a power-law creep solid using indentation tests // Mech. Time-Depend. Mater., 2007. vol.11, no. 2. pp. 159-172. doi: 10.1007/ s11043-007-9033-6.

23. Naumenko K., Altenbach H., Gorash Y. Creep Analysis with a Stress Range Dependent Constitutive Model// Arch. Appl. Mech., 2009. vol.79, no.6. pp. 619-630. doi:10.1007/ s00419-008-0287-5.

24. Радченко В. П. Об одной структурной реологической модели нелинейно-упругого материала // Прикладная механика, 1990. Т. 26, №6. С. 67-74.

25. Радченко В. П., Шапиевский Д. В. Анализ нелинейной обобщенной модели Максвелла // Вестн. Сам. гос. техн. ун-та. Сер. Физ.-мат. науки, 2005. №38. С. 55-64. doi: 10.14498/vsgtu372.

26. Радченко В. П., Шапиевский Д. В. О дрейфе упругой деформации для нелинейноупругих материалов вследствие ползучести // Вестн. Сам. гос. техн. ун-та. Сер. Физ.-мат. науки, 2006. №43. С. 99-105. doi: 10.14498/vsgtu458.

27. Радченко В. П., Шапиевский Д. В. Математическая модель ползучести микронеоднородного нелинейно-упругого материала // ПМТФ, 2008. Т. 49, № 3. С. 157-163.

28. Радченко В. П., Андреева Е. А. О дрейфе и эффекте памяти нелинейно-упругой деформации вследствие ползучести для микронеоднородных материалов в условиях одноосного напряженного состояния // Вестн. Сам. гос. техн. ун-та. Сер. Физ.-мат. науки, 2009. № 2(19). С. 72-77. doi : 10.14498/vsgtu712.

29. Хохлов А. В. Определяющее соотношение для реологических процессов: свойства теоретических кривых ползучести и моделирование затухания памяти // Известия РАН. MTT, 2007. № 2. C. 147-166.

30. Хохлов А. В. Определяющее соотношение для реологических процессов с известной историей нагружения. Кривые ползучести и длительной прочности // Изв. PAH. MTT, 2008. № 2. C. 140-160. 
31. Хохлов А. В. Качественный анализ общих свойств теоретических кривых линейного определяющего соотношения вязкоупругости // Наука и образование (МГТУ им. Н. Э. Баумана) (электронный журнал), 2016. №5. C. 187-245. doi: 10.7463/0516. 0840650.

32. Шестериков С. А., Локощенко А. М. Ползучесть и длительная прочность металлов / Итоги науки и техники. Сер. Мех. деформируем. тверд. тела, Т. 13. М.: ВИНИТИ, 1980. C. 3-104.

33. Радченко В. П., Самарин Ю. П. Влияние ползучести на величину упругой деформации слоистого композита // Механика композитных материалов, 1983. Т. 19, № 2. С. 231237.

34. Кнетс И. В., Вилкс Ю. К. Ползучесть компактной костной ткани человека при растяжении // Механика полимеров, 1975. Т. 11, № 4. С. 634-638.

35. Мелнис А. Э., Лайзан Я. Б. Нелинейная ползучесть компактной костной ткани человека при растяжении // Механика полимеров, 1978. Т. 14, №1. С. 97-100.

36. Dandrea J., Lakes R. S. Creep and creep recovery of cast aluminum alloys // Mech. TimeDepend. Mater., 2009. vol. 13, no. 4. pp. 303-315. doi: 10.1007/s11043-009-9089-6.

37. Хохлов А. В. Кривые ползучести и релаксации нелинейного определяющего соотношения Ю. Н. Работнова для вязкоупругопластичных материалов // Проблемы прочности и пластичности, 2016. Т. 78, № 4. С. 452-466.

38. Работнов Ю. Н. Некоторые вопросы теории ползучести // Вестник МГУ, 1948. № 10. C. 81-91.

39. Ильюшин А. А., Победря Б. Е. Основы математической теории термовязкоупругости. М.: Наука, 1970. 280 с.

40. Виноградов Г. В., Малкин А. Я. Реология полимеров. М.: Химия, 1977. 440 с.

41. Коларов Д., Балтов А., Бончева Н. Механика пластических сред. М.: Мир, 1979. 304 с.

42. Кайбышев О. А. Сверхпластичность промышленных сплавов. М.: Металлургия, 1984. 264 c.

43. Brinson H. F., Brinson L. C. Polymer Engineering Science and Viscoelasticity. Berlin: Springer Science, 2008. xvi+446 pp. doi : 10.1007/978-0-387-73861-1.

44. Bergstrom J. S. Mechanics of Solid Polymers. Theory and Computational Modeling. Amsterdam: William Andrew is an imprint of Elsevier, 2015. xiv+509 pp. doi: 10.1016/ c2013-0-15493-1.

45. Lin Y. C., Chen X.-M. A critical review of experimental results and constitutive descriptions for metals and alloys in hot working// Materials and Design, 2011. vol. 32, no. 4. pp. 1733-1759. doi: 10.1016/j.matdes.2010.11.048.

46. Lee W.-S., Lin C.-R. Deformation behavior and microstructural evolution of 7075-T6 aluminum alloy at cryogenic temperatures// Cryogenics, 2016. vol.79. pp. 26-34. doi: 10 . 1016/j.cryogenics.2016.07.007. 
MSC: 74D10

\title{
The nonlinear Maxwell-type model for viscoelastoplastic materials: simulation of temperature influence on creep, relaxation and strain-stress curves
}

\author{
A. V. Khokhlov \\ Lomonosov Moscow State University, \\ Institute of Mechanics, \\ 1, Michurinsky prospekt, Moscow, 119192, Russian Federation.
}

\begin{abstract}
The nonlinear Maxwell-type constitutive relation with two arbitrary material functions for viscoelastoplastic multi-modulus materials is studied analytically in uniaxial isothermic case to reveal the model abilities and applicability scope and to develop techniques of its identification, tuning and fitting. The constitutive equation is aimed at adequate modeling of the rheological phenomena set which is typical for reonomic materials exhibiting non-linear hereditary properties, strong strain rate sensitivity, secondary creep, yielding at constant stress, tension compression asymmetry and such temperature effects as increase of material compliance, strain rate sensitivity and rates of dissipation, relaxation, creep and plastic strain accumulation with temperature growth. The model is applicable for simulation of mechanical behaviour of various polymers, their solutions and melts, solid propellants, sand-asphalt concretes, composite materials, titanium and aluminum alloys, ceramics at high temperature and so on.

To describe the influence of temperature on material mechanical behavior (under isothermic conditions), two scalar material parameters of the model (viscosity coefficient and "modulus of elasticity") are considered as a functions of temperature level. The general restrictions on their properties which are necessary and sufficient for adequate qualitative description of the basic thermomechanical phenomena related to typical temperature influence on creep and relaxation curves, creep recovery curves, creep curves under stepwise loading and quasi-static stress-strain curves of viscoelastoplastic materials are obtained. The restrictions are derived using systematic analytical study of general qualitative features of the theoretic creep and relaxation curves, creep curves under step-wise loading, long-term strength curves and stress-strain curves at constant strain or stress rates generated by the constitutive equation (under minimal restrictions on material functions) and their comparison to typical test curves of stable viscoelastoplastic materials. It is
\end{abstract}

\footnotetext{
Article

๖ (ㅇ)(7) The content is published under the terms of the Creative Commons Attribution 4.0 International License (http://creativecommons.org/licenses/by/4.0/)

Please cite this article in press as:

Khokhlov A. V. The nonlinear Maxwell-type model for viscoelastoplastic materials: simulation of temperature influence on creep, relaxation and strain-stress curves, Vestn. Samar. Gos. Tekhn. Univ., Ser. Fiz.-Mat. Nauki [J. Samara State Tech. Univ., Ser. Phys. Math. Sci.], 2017, vol. 21, no. 1, pp. 160-179. doi: 10.14498/vsgtu1524 (In Russian).
}

Author's Details:

Andrew V. Khokhlov (1) http://orcid.org/0000-0002-9212-2579

Cand. Techn. Sci.; Senior Researcher; Lab. of Elasticity and Plasticity;

e-mail: andrey-khokhlov@ya.ru 
proved that the viscosity coefficient and the "modulus of elasticity" of the model and their ratio (i.e. relaxation time of the associated linear Maxwell model) should be decreasing functions of temperature. This requirements are proved to provide an adequate qualitative simulation of a dozen basic phenomena expressing an increase of material compliance (a decrease of tangent modulus and yield stress, in particular), strengthening of strain rate sensitivity and acceleration of dissipation, relaxation, creep and plastic strain accumulation with temperature growth.

Keywords: nonlinear viscoelastoplasticity, isothermic conditions, influence of temperature, relaxation curves, creep curves, step-wise loadings, stressstrain curves, yield stress, rate sensitivity, superplasticity, ratcheting, polymers.

Received: $6^{\text {th }}$ December, $2016 /$ Revised: $6^{\text {th }}$ March, $2017 /$ Accepted: $13^{\text {th }}$ March, $2017 /$ First online:

Competing interests. I have no competing interests.

Author's Responsibilities. I take full responsibility for submitting the final manuscript in print. I approved the final version of the manuscript.

Funding. This work was supported by the Russian Foundation for Basic Research (project no. 17-08-01146_a).

\section{References}

1. Khokhlov A. V. Properties of stress-strain curves generated by the nonlinear Maxwell-type viscoelastoplastic model at constant stress rates, Mashinostroenie $i$ inzhenernoye obrazovanie, 2017, no. 1, pp. 14-28 (In Russian).

2. Khokhlov A. V. Properties of the nonlinear Maxwell-type model with two material functions for viscoelastoplastic materials, Moscow University Mechanics Bulletin. Ser.1. Matematica, mekhanica, 2016, no. 6, pp. 36-41 (In Russian).

3. Khokhlov A. V. The nonlinear Maxwell-type viscoelastoplastic model: properties of creep curves at piecewise-constant stress and the criterion for plastic strain accumulation, Mashinostroenie i inzhenernoe obrazovanie, 2016, no. 3, pp. 35-48 (In Russian).

4. Khokhlov A. V. Long-term strength curves generated by the nonlinear Maxwell-type model for viscoelastoplastic materials and the linear damage rule under step loading, Vestn. Samar. Gos. Tekhn. Univ. Ser. Fiz.-Mat. Nauki [J. Samara State Tech. Univ., Ser. Phys. Math. Sci.], 2016, vol. 20, no. 3, pp. 524-543. doi: 10.14498/vsgtu1512.

5. Il'yushin A. A., Ogibalov P. M. A certain generalization of the Voigt and Maxwell models, Polymer Mechanics, 1966, vol.2, no. 2, pp. 122-124. doi: 10.1007/BF00867097.

6. Gorodtsov V. A., Leonov A. I. On the kinematics, nonequilibrium thermodynamics, and rheological relationships in the nonlinear theory of viscoelasticity, J. Appl. Math. Mech., 1968, vol. 32, no. 1, pp. 62-84. doi: 10.1016/0021-8928(68)90148-2.

7. Leonov A. I., Lipkina E. Ch., Paskhin E. D., Prokunin A. N. Theoretical and experimental investigations of shearing in elastic polymer liquids, Rheol. Acta, 1976, vol. 15, no. 7/8, pp. 411-426. doi: 10.1007/BF01574496.

8. Pal'mov V. A. Rheological models in the nonlinear mechanics of deformable bodies, Uspekhi mekhaniki, 1980, vol. 3, no. 3, pp. 75-115 (In Russian).

9. Prokunin A. N. On the non-linear Maxwell-type defining equations for describing the motions of polymer liquids, J. Appl. Math. Mech., 1984, vol.48, no.6, pp. 699-706. doi: 10.1016/0021-8928(84)90037-6. 
10. Larson R. G. Constitutive Equations for Polymer Melts and Solutions. Boston, Butterworth, 1988, 364 pp. doi: 10.1016/c2013-0-04284-3.

11. Leonov A. I. Analysis of simple constitutive equations for viscoelastic liquids, Journal of Non-Newtonian Fluid Mechanics, 1992, vol.42, no.3, pp. 323-350. doi:10.1016/ 0377-0257 (92)87017-6.

12. Leonov A. I., Prokunin A. N. Non-linear Phenomena in Flows of Viscoelastic Polymer Fluids. London, Chapman and Hall, 1994, xvii+475 pp. doi : 10.1007/978-94-011-1258-1.

13. Leonov A. I. Constitutive equations for viscoelastic liquids: Formulation, analysis and comparison with data, Rheology Series, 1999, vol. 8, pp. 519-575. doi : 10.1016/S0169-3107 (99) 80040-9.

14. Kremple E., Ho K. Inelastic Compressible and Incompressible, Isotropic, Small Strain Viscoplasticity Theory Based on Overstress (VBO), In: Handbook of Materials Behavior Models. New York, Academic Press, 2001, pp. 336-348. doi : 10.1016/b978-012443341-0/50037-5.

15. Rabotnov Yu. N. Creep problems in structural members. Amsterdam, London, NorthHolland Publ. Co., 1969, xiv+822 pp.

16. Malinin N. N. Raschety na polzuchest' elementov mashinostroitel'nykh konstruktsii [Computations of Creeping of Engineering Construction Elements]. Moscow, Mashinostroenie, 1981, 221 pp. (In Russian)

17. Betten J. Creep Mechanics. Berlin, Heidelberg, Springer-Verlag, 2008, xiii +353 pp. doi : 10 . $1007 / \mathrm{b} 138749$.

18. Lokoshchenko A. M. Polzuchest' $i$ dlitel'naia prochnost' metallov [Creep and Long-Term Strength of Metals]. Moscow, Fizmatlit, 2016, 504 pp. (In Russian)

19. Vasin R. A., Enikeev F. U. Introduction in mechanics of superplasticity. Ufa, Gilem, 1998, 280 pp. (In Russian)

20. Nieh T. G., Wadsworth J., Sherby O. D. Superplasticity in metals and ceramics, Cambridge Solid State Science Series. Cambridge, Cambridge University Press, 1997, xiv +273 pp. doi: $10.1017 /$ CB09780511525230.

21. Segal V. M., Beyerlein I. J., Tome C. N., Chuvil'deev V. N., Kopylov V. I. Fundamentals and Engineering of Severe Plastic Deformation. New York, Nova Science Pub. Inc., 2010, $542 \mathrm{pp}$.

22. Cao Y. Determination of the creep exponent of a power-law creep solid using indentation tests, Mech. Time-Depend. Mater., 2007, vol.11, no.2, pp. 159-172. doi:10.1007/ s11043-007-9033-6.

23. Naumenko K., Altenbach H., Gorash Y. Creep Analysis with a Stress Range Dependent Constitutive Model, Arch. Appl. Mech., 2009, vol.79, no.6, pp. 619-630. doi:10.1007/ s00419-008-0287-5.

24. Radchenko V. P. Structural rheological model of a nonlinearly elastic material, Soviet Applied Mechanics, 1990, vol. 26, no.6, pp. 577-582. doi : 10.1007/BF00887589.

25. Radchenko V. P., Shapievskii D. V. Analysis of a nonlinear generalized Maxwell model, Vestn. Samar. Gos. Tekhn. Univ., Ser. Fiz.-Mat. Nauki [J. Samara State Tech. Univ., Ser. Phys. Math. Sci.], 2005, no. 38, pp. 55-64 (In Russian). doi: 10.14498/vsgtu372.

26. Radchenko V. P., Shapievskii D. V. Drift of elastic deformation due to creep for nonlinear elastic materials, Vestn. Samar. Gos. Tekhn. Univ. Ser. Fiz.-Mat. Nauki [J. Samara State Tech. Univ., Ser. Phys. Math. Sci.], 2006, no. 43, pp. 99-106 (In Russian). doi: 10.14498/ vsgtu458.

27. Radchenko V. P., Shapievskii D. V. Mathematical model of creep for a microinhomogeneous nonlinearly elastic material, J. Appl. Mech. Tech. Phys., 2008, vol.49, no. 3, pp. 478-483. doi: 10.1007/s10808-008-0064-9.

28. Radchenko V. P., Andreeva E. A. On drift and memory effect of nonlinear elastic strain arising due to creep for micronon-uniform materials in conditions of linear stress, Vestn. Samar. Gos. Tekhn. Univ. Ser. Fiz.-Mat. Nauki [J. Samara State Tech. Univ., Ser. Phys. Math. Sci.], 2009, no. 2(19), pp. 72-77 (In Russian). doi: 10.14498/vsgtu712. 
29. Khokhlov A. V. Constitutive relation for rheological processes: Properties of theoretic creep curves and simulation of memory decay, Mech. Solids, 2007, vol.42, no. 2, pp. 291-306. doi: $10.3103 / \mathrm{S} 0025654407020148$.

30. Khokhlov A. V. Constitutive relation for rheological processes with known loading history. Creep and long-term strength curves, Mech. Solids, 2008, vol. 43, no. 2, pp. 283-299. doi : 10. 3103/S0025654408020155.

31. Khohlov A. V. The Qualitative Analysis of Theoretic Curves Generated by Linear Viscoelasticity Constitutive Equation, Science and Education of the Bauman MSTU, 2016, no. 5, pp. 187-245 (In Russian). doi : 10.7463/0516.0840650.

32. Shesterikov S. A., Lokoshchenko A. M. Creep and long-term strength of metals, Itogi nauki i tekhniki. Ser. Mekh. deformiruem. tverd. tela [Achievements in Science and Technology. Ser. Mechanics of Solids], 13. Moscow, VINITI, 1980, pp. 3-104 (In Russian).

33. Radchenko V. P, Samarin Yu. P. Effect of creep on the elastic deformation of a laminar composite, Mech. Compos. Mater., 1983, vol. 19, no. 2, pp. 162-168. doi: 10.1007/BF00604218.

34. Knets I. V., Vilks Yu. K. Creep of compact human bony tissue under tension, Polymer Mechanics, 1975, vol. 11, no. 4, pp. 543-547. doi: 10.1007/BF00856779.

35. Melnis A. É., Laizan Ya. B. Nonlinear creep of human compact bone tissue upon stretching, Polymer Mechanics, 1978, vol. 14, no. 1, pp. 82-84. doi: 10.1007/BF00859565.

36. Dandrea J., Lakes R. S. Creep and creep recovery of cast aluminum alloys, Mech. TimeDepend. Mater., 2009, vol. 13, no. 4, pp. 303-315. doi:10.1007/s11043-009-9089-6.

37. Khokhlov A. V. Creep and relaxation curves produced by the Rabotnov nonlinear constitutive relation for viscoelastoplastic materials, Problemy prochnosti i plastichnosti, 2016, vol. 78, no. 4, pp. 452-466 (In Russian).

38. Rabotnov Yu. N. Some questions of the creep theory, Vestnik MGU, 1948, no. 10, pp. 81-91 (In Russian).

39. Il'iushin A. A., Pobedrya B. E. Osnovy matematicheskoi teorii termoviazkouprugosti [Fundamentals of the Mathematical Theory of Thermoviscoelasticity]. Moscow, Nauka, 1970, 280 pp. (In Russian)

40. Vinogradov G. V., Malkin A. Ya. Rheology of Polymers. Viscoelasticity and Flow of Polymers. Berlin, Springer-Verlag, 1980, xii+468 pp. doi : 10.1007/978-3-642-52204-8.

41. Kolarov D., Baltov A., Boncheva N. Mekhanika plasticheskikh sred [Mechanics of Plastic Media]. Moscow, Mir, 1979, 304 pp. (In Russian)

42. Kaibyshev O. A. Sverkhplastichnost' promyshlennykh splavov [Superplasticity of industrial alloys]. Moscow, Metallurgiia, 1984, 264 pp. (In Russian)

43. Brinson H. F., Brinson L. C. Polymer Engineering Science and Viscoelasticity. Berlin, Springer Science, 2008, xvi+446 pp. doi : 10.1007/978-0-387-73861-1.

44. Bergstrom J. S. Mechanics of Solid Polymers. Theory and Computational Modeling. Amsterdam, William Andrew is an imprint of Elsevier, 2015, xiv+509 pp. doi: 10.1016/ c2013-0-15493-1.

45. Lin Y. C., Chen X.-M. A critical review of experimental results and constitutive descriptions for metals and alloys in hot working, Materials and Design, 2011, vol.32, no. 4, pp. 1733-1759. doi: 10.1016/j.matdes.2010.11.048.

46. Lee W.-S., Lin C.-R. Deformation behavior and microstructural evolution of 7075-T6 aluminum alloy at cryogenic temperatures, Cryogenics, 2016, vol.79, pp. 26-34. doi: 10.1016/ j.cryogenics.2016.07.007. 